\title{
THE EFFECT OF AMPHIPHILIC LIQUID CRYSTALLINE SOLVENTS ON THE HYDROLYSIS OF BENZYLIDENE t-BUTYLAMINE N-OXIDE $\left(^{*}\right)$
}

\author{
W. E. BACON and J. W. THOMAS \\ Liquid Crystal Institute, Kent State University, Kent, Ohio 44242, U.S.A.
}

\begin{abstract}
The rate of hydrolysis of benzylidene t-butylamine N-oxide decreases as the concentration of dodecylammonium chloride in water increases until molar concentrations are reached. At these concentrations a maximum inhibition in the rate of hydrolysis is reached which corresponds to the formation of the lamellar mesophase. This maximum inhibition, caused by the lamellar structure, appears to be due to long-range electrostatic effects upon the protonated intermediates of the reaction.
\end{abstract}

1. Introduction. - Anisotropic media orient solute molecules in solution altering rates of reaction, stereochemistry and the ratio of products [1]. A major part of the studies investigating the role of anisotropic media on chemical reactions utilize thermotropic liquid crystalline media and only a few investigations are reported with lyotropic liquid crystalline solvents $[2,3]$. Furthermore, most of the studies reported include buffer systems within the lyotropic media that may be responsible for altering the texture of the liquid crystalline media.

The organization of amphiphilic molecules in aqueous solution encompasses small micellar forms or large aggregates of liquid crystalline structures depending upon the concentration of the amphiphile. The role of micellar forms in aqueous solution on chemical reactions is well known [4]. We wish to report a study of the influence of lyotropic liquid crystalline mesophases on acid hydration reactions in the absence of buffers within the media. The acidity of the media does not change significantly during the reaction because small samples of substrate can be used and the reaction can be followed spectrophotometrically. The initial reaction for study is the hydrolysis of benzylidene $\mathrm{t}$-butylamine $\mathrm{N}$-oxide, $\mathrm{I}$, in aqueous solutions of dodecylammonium chloride, DdAC, at concentrations ranging from above critical micelle concentration through $1.6 \mathrm{M}$ or the liquid crystalline mesophases

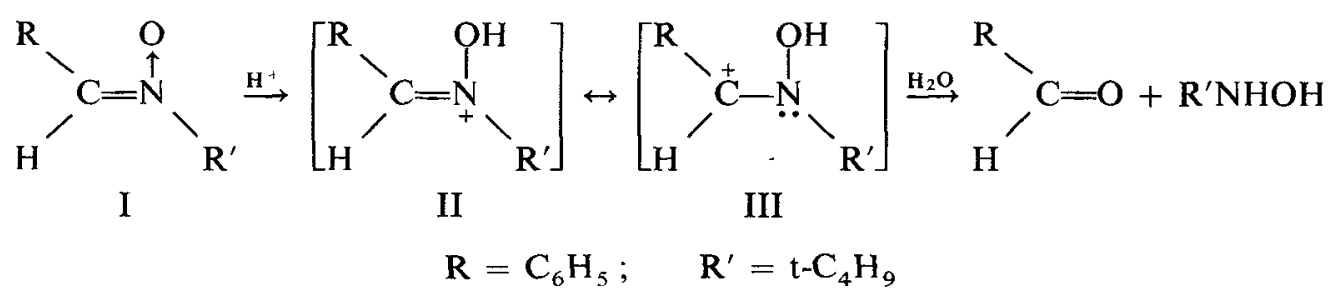

This reaction was selected for study because cationic surfactants inhibit acid hydration reactions [5] possibly through a long-range electrostatic effect originating

(*) This work was supported by the National Science Foundation under grant DMR76-21339. from the charged micelle interface [6]. The lamellar neat soap phase, for example, is reported to have a conductivity similar to that of a fused salt and the middle soap (hexagonal) phase even greater than the lamellar phase [7]. We think aggregate structures of the lamellar or hexagonal packed liquid crystalline 
structures may show a maximum electrostatic effect on these reactions and therefore a maximum inhibition.

2. Experimental. - Benzylidene t-butylamine Noxide, $\mathrm{I}$, was prepared in $72 \%$ yield by the thermal isomerization of 2-t-butyl-3-phenyloxaziridine according to a procedure reported by Emmons [8]. The latter compound was prepared from the corresponding Schiff base $[8,9]$ by oxidation with $\mathrm{m}$-chloroperbenzoic acid as reported by Pews [10]. $\mathrm{I}$, was recrystallized from $\mathrm{n}$-hexane, $\mathrm{mp} 72-3^{\circ}$, and the nmr spectra compared with the same compound used in previous studies [9].

Dodecylammonium chloride, DdAC was prepared by the procedures reported $[11,12]$ and the aqueous solutions were prepared from the recrystallized salt and distilled water just before each rate determination. The $\mathrm{pH}$ of all solutions was adjusted by the addition of conc. hydrochloric acid at $25^{\circ}$ using a Fisher Accumet $\mathrm{pH}$ Meter with an expanded scale, equipped with a Corning combination electrode. Standard buffers were used to calibrate the $\mathrm{pH}$ meter before each rate was determined. Prolonged exposure of the glass electrode to the surfactant solution caused a slow response of the electrode to the $\mathrm{pH}$ of the solution. The electrode response could be restored by soaking in $0.1 \mathrm{M} \mathrm{HCl}$. The hydrolysis of $\mathrm{I}$, was followed by the consumption of reactant at $292 \mathrm{~nm}$ in thermostatted cells, at $25.0 \pm 0.05^{\circ}$, with a G. C. A. McPherson Eu 700 series recording spectrophotometer equipped with an automatic filter attachment. The reaction was initiated by the injection of 9 microliters of an aqueous solution of $I$, into a cuvette and then stirred to assure homogeneous solution. The pseudo first-order plots were linear to greater than $90 \%$ reaction and the reproductibility is within $5 \%$.

3. Results and discussion. - The rate of hydrolysis of $\mathrm{I}$, is increased in the presence of the anionic surfactant, sodium lauryl sulfate, NaLS, however, at CMC the cationic amphiphile, hexadecyltrimethylammonium bromide, CTAB, had virtually no effect on the rate [13]. The catalytic effect by the former surfactant was explained as a consequence of electrostatic interactions between the positive charged intermediate and the negative charged surface of the NaLS micelle and the absence of a catalytic reaction by the latter is consistent with the electrostatic effect [13].

The rate of hydrolysis of $\mathrm{I}$, is inhibited by the cationic surfactant, DdAC as shown in figure 1. The observed pseudo first-order rate constant decreases as the concentration of DdAC increases until one molar concentration is reached then the rate tends to level off. The change in the rate of hydrolysis in solutions of n-butylammonium chloride at comparable concentrations is much less as shown in figure 1. Although an increase in the cation and

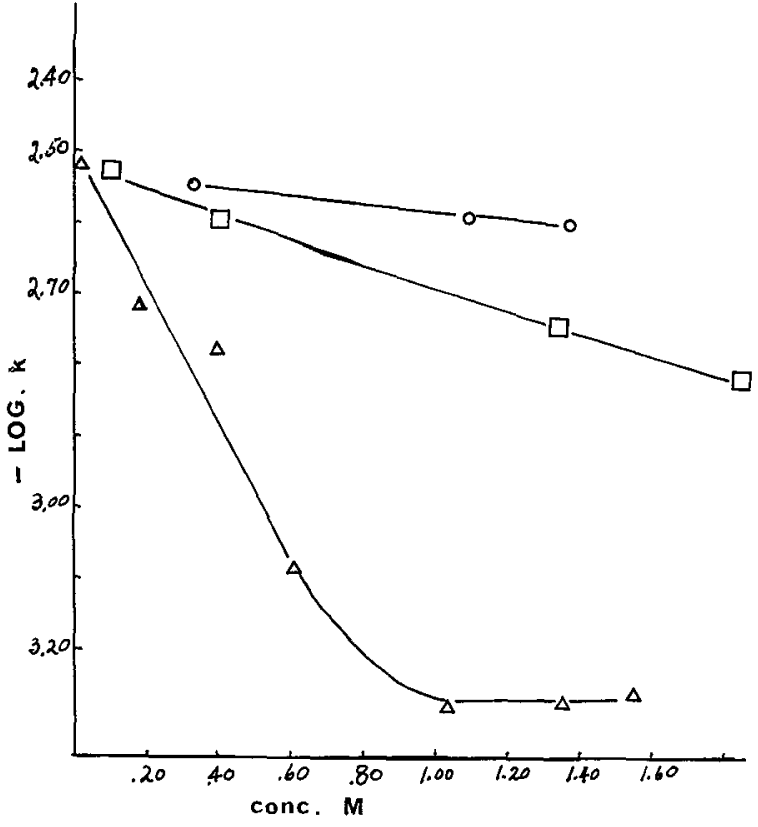

Fig. 1. - Pseudo first-order rate constant of I, versus the concentration of electrolyte in solution at $\mathrm{pH} 1.00 . \triangle$ rate observed in DdAC solutions; $\square$ n-butylammonium chloride; and $\mathrm{O} N \mathrm{NaCl}$ solutions.

anion concentrations in aqueous solution shows some inhibition in the rate of hydrolysis of $\mathrm{I}$, it does not appear to explain the effect of DdAC on the hydrolysis reaction. Comparable concentrations of sodium chloride in aqueous solution inhibits the hydrolysis of $\mathrm{I}$, less than n-butylammonium chloride solutions.

A mechanism for the acid hydrolysis of $\mathrm{I}$, in aqueous and micellar solutions is proposed $[9,13]$, however, rate studies at high concentrations, i.e. molar, of cationic aqueous surfactant solutions has not been reported. Previous work reports a linear relationship when the log of the rate of hydrolysis of $I$, is plotted as a function of $\mathrm{pH}$ in aqueous solutions [9]. We have observed a similar linear plot with $1.36 \mathrm{M}$ aqueous DdAC solution and this is shown in figure 2.

In solutions where the concentration of electrolyte was varied the $\mathrm{pH}$ was adjusted to 1.00 before determining the rate of hydrolysis of $\mathrm{I}$, and with substrate concentrations of $10^{-5} \mathrm{M}$ no change in $\mathrm{pH}$ could be detected after hydrolysis was complete. The curve shown in figure 1 , for the hydrolysis in DdAC solutions forms a plateau in the region of about one molar concentration and the evidence from microscopic investigation of the three solutions above molar concentrations indicate the presence of liquid crystalline mesophases. We were unable to detect evidence of mesophases in less than molar concentrations of aqueous DdAC solutions used in this study ; suggesting the inhibition of the rate of hydrolysis of $I$, reaches a stationary state with the formation of the liquid crystalline mesophase. Extension of 


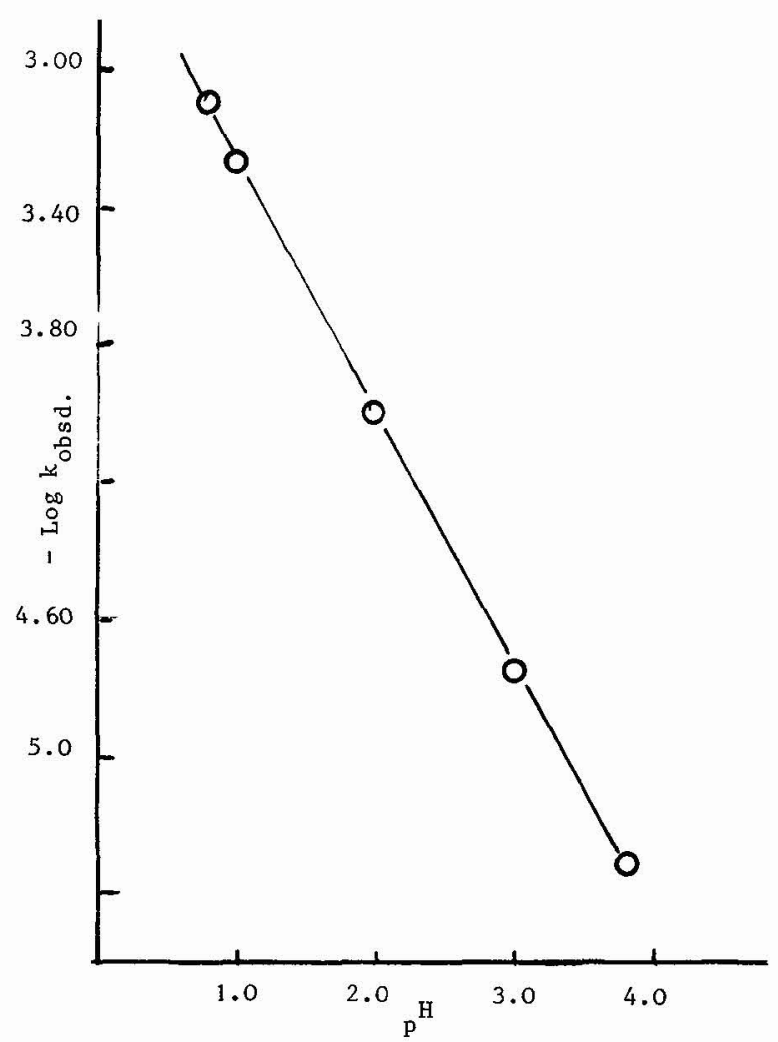

Fig. 2. - Pseudo first-order rate constant of I, dissolved in $1.36 \mathrm{M}$ aqueous DdAC as a function of $\mathrm{pH}$.

this study to $1.89 \mathrm{M}$ aqueous DdAC solutions at $\mathrm{pH}$ 1.00 failed because of a solid phase separation at $25^{\circ}$. If the inhibition of the rate of hydrolysis of 1 , is the consequence of electrostatic repulsion between the positively charged protonated intermediate II or III and the cationic liquid crystalline structures in solution then a maximum electrostatic effect is developed in solutions with above molar concentrations where liquid crystalline structures are formed. In contrast the rate of hydrolysis in solutions of comparable concentration of n-butylammonium chloride is inhibited much less than in DdAC solutions, perhaps because these solutions do not contain molecular aggregates and do not appear anisotropic at the concentrations used in this work. The inhibition of hydrolysis of $I$, is less in sodium chloride solutions than in n-butylammonium chloride or DdAC and this probably reflects the cationic electrostatic effect because in all cases the rate of hydrolysis is less than in aqueous hydrochloric acid solution at the same $\mathrm{pH}$.

An alternate explanation for the inhibition of acid hydration reactions by cationic micelles is based on the assumption that the substrate is partitioned between water and the micelles but that hydrogen ions are excluded from the micelles [5]. This may be an alternate explanation for the inhibition of the hydrolysis of $\mathrm{I}$, in DdAC solutions, however, we would not expect the rate of hydrolysis to level off as shown in figure 1. Additional study is underway to find the microenvironment of the substrate in liquid crystalline solutions before we can suggest a mechanism for the levelling-off of the rate of hydrolysis.

The presence of liquid crystalline mesophases in aqueous DdAC solutions at concentrations greater than $35 \%$ by weight are reported $[11,14]$ however,

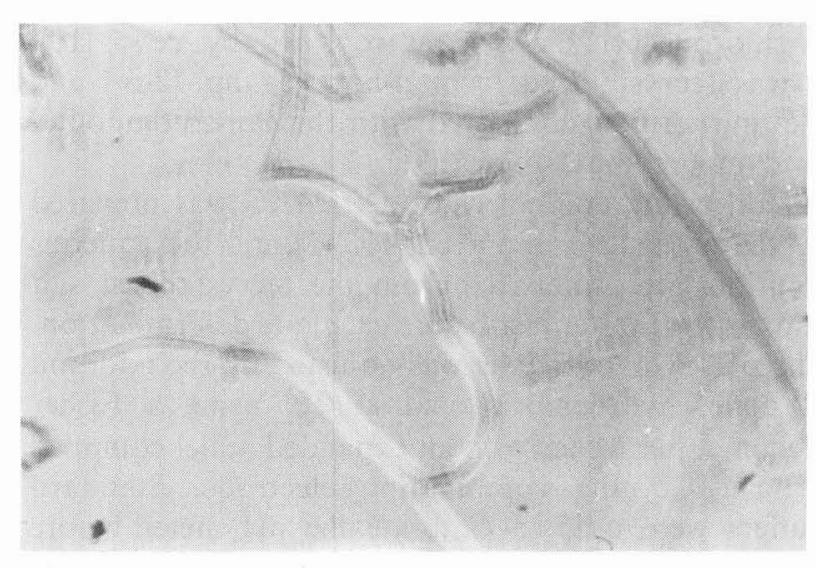

a)

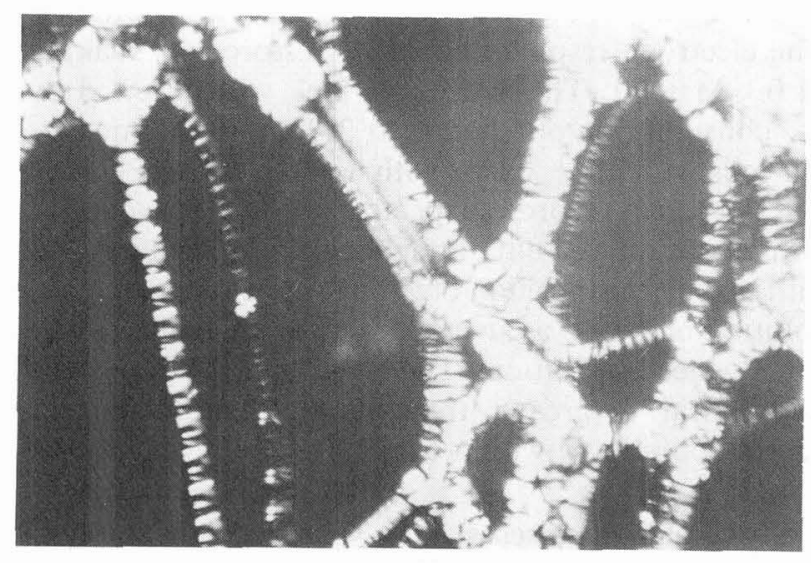

b)

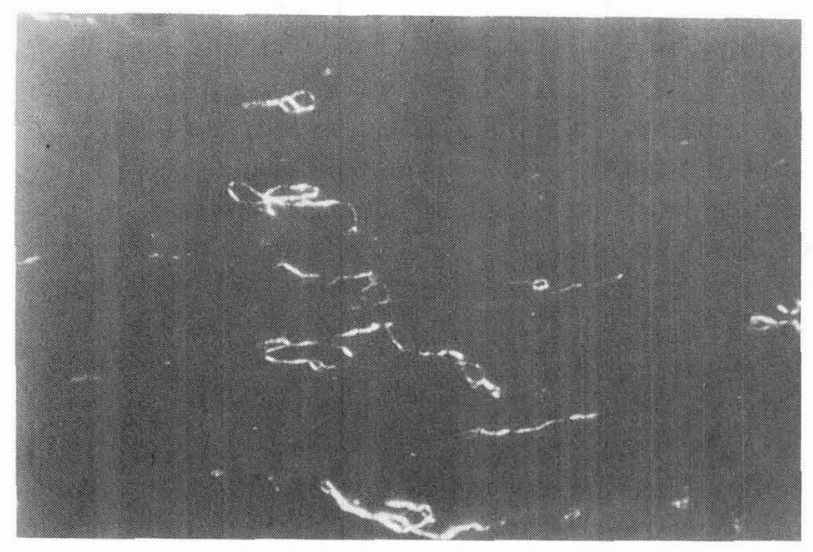

c)

FIG. 3. - Oily streaks and pseudoisotropic texture of lamellar solutions; pH 1.00; crossed polarizers, 96x. a) $1.36 \mathrm{M}$ aqueous DdAC ; b) $1.54 \mathrm{M}$ aqueous DdAC; $c$ ) $1.04 \mathrm{M}$ aqueous DdAC. 
the classification of the types of mesophases was not completed. Recently a solution of $31.2 \%$ by weight of DdAC dissolved in $1 \%$ aqueous hydrochloric acid solution (by weight) was reported to show a well defined lamellar to nematic transition at $32^{\circ}$, followed at $50.9^{\circ}$ by an isotropic [15]. We prepared a solution of the same composition; adjusted to $\mathrm{pH} 1.00$, and this solution is represented as the $1.36 \mathrm{M}$ DdAC solution in figure 1. Microscopic investigation of this solution shows the presence of oily streaks and a pseudoisotropic texture indicative of the lamellar mesophase (Fig. 3). This observation is in accord with the lamellar mesophase reported for the solution of $31.2 \%$ of DdAC dissolved in $1 \%$ hydrochloric Acid [15].

The mobility of the $1.56 \mathrm{M}$ and the $1.36 \mathrm{M}$ solutions are about the same and more mobile than the middle phase of most soaps. Similar microscopic evidence for the $1.56 \mathrm{M}$ solution also suggests the presence of the lamellar mesophase as shown in figure $3 b$. Although the oily streaks shown in figure $3 a$ exhibit a fine cross-banding the latter streaks contain a coarser focal conic texture with the higher index component vibrating crosswise [17].

The point in the plateau area of the DdAC curve corresponding to the $1.04 \mathrm{M}$ solution in figure 1 , contains more water than the other two points suggest- ing perhaps a middle phase may be formed. Increasing the water content frequently causes a phase transition from the lamellar to the hexagonal phase with cylindrical aggregates [16]. The polar head groups of the surfactant molecules compose the surface of the cylinders which are arranged in a hexagonal packing scheme with water between the aggregates. This arrangement is expected to exert a strong electrostatic effect upon the hydrolysis of I. Observations of the 1.04 DdAC solution also suggest the lamellar phase based upon the oily streaks, pseudo-isotropic texture shown in figure $3 c$ and the mobility of the solution, however, we are continuing observations in this concentration range.

In conclusion, it appears the lamellar mesophase exerts a maximum electrostatic effect upon the protonated intermediates, II or III, thereby causing a maximum inhibition of the rate of hydrolysis as the concentration of DdAC reaches one molar. However, the validity of this conclusion must await the determination of the reaction site in the liquid crystalline mesophase.

Acknowledgment. - The authors would like to thank Dr. Mark Greenberg and Dr. Keith Radley for the many helpful discussions and the National Science Foundation for financial support.

\section{References}

[1] Bacon, W. E., J. Physique Colloq. 36 (1975) C1-409.

[2] Ahmad, S. I. and Friberg, S., J. Amer. Chem. Soc. 94 (1972) 5196.

[3] Murthy, K. S. and RiPPIE, E. G., J. Pharm. Science 59 (1970) 459.

[4] Fendler, J. H. and Fendler, E. J., Catalysis in Micellar and Macromolecular Systems (Academic Press, New York) 1975.

[5] Bunton, C. A., Rivera, F. and Sepulveda, L., J. Org. Chem. 43 (1978) 1166.

[6] Menger, F. M., Portnoy, C. E., J. Amer. Chem. Soc. 89 (1967) 4698.

[7] Gray, G. W. and Winsor, P. A., Eds., Liquid Crystals and Plastic Crystals (Horwood, Chichester, England) 2 (1974) p. 125 .

[8] Emmons, W. D., J. Amer. Chem. Soc. 79 (1957) 5739.

[9] O'Connor, C. J., Fendler, E. J. and Fendier, J. H., J. Chem. Soc., Perkin II (1973) 1744.
[10] Pews, R. G., J. Org. Chem. 32 (1967) 1628.

[11] Ralston, A. W., Hoffman, E. J., Hoern, W. and Selby, W. M., J. Amer. Chem. Soc. 63 (1941) 1598.

[12] Nishikido, N., J. Colloid Interface Sci. 60 (1977) 242.

[13] O’Connor, C. J., Fendler, E. J. and Fendler, J. H., J. Chem. Soc., Perkin II (1973) 1900.

[14] Broome, F. K., Hoern, C. W. and Harwood, H. J., J. Amer. Chem. Soc. 73 (1951) 3350.

[15] Andrews, J. T. S., Radley, K. and SAupe, A., Abstracts Sixth International Liquid Crystal Conference, Kent State University, 1976.

[16] SAUPE, A., Annual Reviews of Physical Chemistry 24 (1973) 441, Palo Alto, Calif. U.S.A.

[16] Saupe, A., Annual Reviews of Physical Chemistry, Palo Alto, Calif. U.S.A. 24 (1973) 441.

[17] Rosevear, F. B., J. Amer. Oil Chem. Soc. 31 (1954) 628. 